\title{
Current Algebra and Bosonization in Three Dimensions
}

\author{
J.C. Le Guillou ${ }^{a *}$, C. Núñez ${ }^{b}$ and F.A. Schaposnik ${ }^{a \dagger \ddagger}$ \\ ${ }^{a}$ Laboratoire de Physique Théorique ENSLAPP $\S$ \\ LAPP, B.P. 110, F-74941 Annecy-le-Vieux Cedex, France \\ ${ }^{b}$ Departamento de Física, Universidad Nacional de La Plata \\ C.C. 67, 1900 La Plata, Argentina
}

\begin{abstract}
We consider the fermion-boson mapping in three dimensional space-time, in the Abelian case, from the current algebra point of view. We show that in a path-integral framework one can derive a general bosonization recipe leading, in the bosonic language, to the correct equal-time current commutators of the original free fermionic theory.
\end{abstract}

*Also at Université de Savoie and at Institut Universitaire de France

$\dagger$ Investigador CICBA, Argentina

${ }^{\ddagger}$ On leave from Universidad Nacional de La Plata, Argentina

$\S$ URA 1436 du CNRS associée à l'Ecole Normale Supérieure de Lyon et latex courà l'Université de Savoie 


\section{Introduction}

The mapping of two-dimensional fermionic quantum field theories onto equivalent bosonic quantum field theories [1]-[5], commonly called bosonization, can be viewed as the consequence of a non-trivial current algebra. In particular, difficulties in finding the non-Abelian bosonization recipe were overcome when the relevance of current algebra was put forward. Indeed, the equivalence of the theory of $N$ massless-free fermions and the $S O(N)$ WessZumino-Witten model was proven by showing that the Kac-Moody and Virasoro algebras coincide in the two theories [5].

Problems encountered in trying to extend bosonization to $d>2$ dimensions can be thought as the result, in part, of a much more complicated algebraic structure of current algebra. In particular, Schwinger terms [6][8] which arise when computing equal time fermion current commutators are divergent in $d>2$ dimensions [9]-[11], in contrast with the finite $d=2$ result.

In spite of these difficulties and without making appeal to current algebra, a fermion-boson mapping has been established in $d=3$ dimensions [12]-[22]. The resulting bosonization recipes for fermion currents, valid in different regimes, either for free or interacting models, both in the Abelian and nonAbelian cases are the natural extension of the $d=2$ ones [18]-21]. However, a detailed analysis of the relation between fermionic and bosonic current algebra is lacking. We shall address to this issue in the present work.

As we shall see, one can establish using the path-integral approach a fermion-boson mapping leading to an exact bosonization recipe for fermion currents in the Abelian case. This is due to the fact, first exploited in [23] for the $d=2$ case, that external-source dependent terms can be factored out very simply within the path-integral approach. In particular, in 3 dimensional (Euclidean) space-time, in the Abelian case, the fermion current bosonizes according to [21]

$$
j_{\mu}=-i \bar{\psi} \gamma_{\mu} \psi \rightarrow \frac{1}{\sqrt{4 \pi}} \epsilon_{\mu \nu \alpha} \partial_{\nu} A_{\alpha}
$$

where $A_{\alpha}$ is a vector field. Now, the exact bosonic Lagrangian equivalent to the original fermionic one is far too complicated to perform exact calculations and it is for this fact that bosonization implies some approximation in $d=3$ dimensions. A possible simplification consists in either neglecting non-quadratic terms in the construction of the bosonic counterpart of a massless Dirac fermion Lagrangian [13], 22] or, alternatively, to perform a 
low energy approximation at some stage of the derivation so that the results are only valid for very massive fermions [18], [21]. While in the former case the resulting effective action looks rather complicated, in the latter one ends with a very simple Maxwell-Chern-Simons (MCS) theory.

MCS theory is very attractive since it exhibits plenty of interesting properties [24] to be welcome both in Particle Physics and in Condensed Matter Physics [25], its connection with 3-dimensional fermionic theories being precisely of interest in this last domain. One should remind however that the equivalence between the free fermion Lagrangian and MCS theories is not exact but only valid in the large fermion-mass region, this meaning the large-distances regime for fermion fields. Since current commutators test the short-distance regime, one should not take the MCS gauge-invariant algebra as a starting point to reproduce the fermion current commutators. Indeed, the commutator algebra obeyed by the electric and magnetic fields in MCS theories has been shown to have finite derivative terms [24], [26]. Were one naively to use the bosonization recipe (四) in a calculation of current commutators, taking the MCS action as the bosonized equivalent to that for free fermions, the correct divergent Schwinger terms would be absent.

In view of the discussion above, the study of three dimensional equal-time fermion current commutators within the bosonization approach needs an approximation scheme valid for an arbitrary (not necessarily large) fermion mass. To handle this problem, we study in the present work a quadratic approximation of the free massive fermion theory following a recent proposal [22]. In this way, we show how the correct equal-time current commutators arise in the bosonic theory, coinciding with those calculated in the original free fermion model [9]-[1], [27]. As it is the case in two dimensions, by showing that the current algebra resulting from the bosonized theory coincides with that of the original fermion model, we go a step further in the obtention of a fermion-boson mapping in 3 dimensions.

The paper is organized as follows. We give in Section 2 a brief review of our bosonization approach for $d \geq 2$ dimensions and then discuss in Section 3 how the equivalent bosonic theory can be approximated to evaluate current commutators in $d=3$. Following the Bjorken-Johnson-Low method we then show that the current commutators obtained according to our bosonization recipe coincide with the original ones arising in the fermionic model. We finally summarize our results in Section 4. 


\section{Bosonization}

We shall describe in this section how bosonization rule (1) can be derived within the path-integral framework. As first stressed in [23, one important ingredient in path-integral bosonization is the introduction of sources which allow to identify the correct bosonic fields and exhibit the exact bosonization recipe for fermion currents. A second ingredient is the introduction of an auxiliary field which allows for obtaining in a very simple way the bosonized action. This approach to bosonization was originally introduced in [16] to study two-dimensional bosonization and then extended to $d>2$ dimensions, both for Abelian and non-Abelian cases [17]-[21]. We here follow the presentation introduced in 21] but emphasizing on the exactness of the bosonization recipe for fermion currents achieved within this approach.

Consider for definiteness the action for a free massive Dirac fermion, coupled to an external source $s_{\mu}$

$$
\mathcal{L}_{F}=\bar{\psi}(\not+m+i \$) \psi
$$

The corresponding partition function in $d$ dimensional Euclidean spacetime reads

$$
Z_{f e r}[s]=\int D \bar{\psi} D \psi \exp \left[-\int d^{d} x \bar{\psi}(\not \partial+m+i ф) \psi\right]
$$

We now perform the following change of fermion variables

$$
\begin{gathered}
\psi=g(x) \psi^{\prime} \\
\bar{\psi}=\bar{\psi}^{\prime} g^{-1}(x) .
\end{gathered}
$$

Here $g(x)=\exp [i \theta(x)]$. Although we shall consider just the case of one Dirac fermion and then $g \in U(1)$, the non-Abelian case can be analogously treated. One can always define for Dirac fermions a path-integral measure invariant under transformation (4). Then, after the change of variables the partition function becomes

$$
Z_{\text {fer }}[s]=\int \mathcal{D} \bar{\psi} \mathcal{D} \psi \exp \left[-\int d^{d} x \bar{\psi}\left(\emptyset+m+g^{-1} \not \partial g+i \$\right) \psi\right] .
$$

(we have omitted primes in the new fermionic variables). It is evident that $i g^{-1} \not \partial g$ in (5) can be thought as a flat connection and can be then replaced 
by a non-trivial gauge field connection $b_{\mu}$ provided a constraint to ensure its flatness is introduced. Then, we can rewrite (5) in the form

$$
\left.Z_{f e r}[s]=\int \mathcal{D} \bar{\psi} \mathcal{D} \psi \mathcal{D} b_{\mu} \delta\left[\epsilon_{\mu_{1} \mu_{2} \ldots \mu_{d}} f_{\mu_{1} \mu_{2}}\right] \exp \left[-\int d^{d} x \bar{\psi}(\not)+m+i \not b+i \not\right) \psi\right]
$$

where

$$
f_{\mu \alpha}=\partial_{\mu} b_{\alpha}-\partial_{\alpha} b_{\mu}
$$

The next step in our derivation is to integrate out fermions so that the partition function becomes

$$
Z_{f e r}[s]=\int D b_{\mu} \operatorname{det}(\not \partial+m+i \not b+i \oint) \delta\left[\epsilon_{\mu_{1} \mu_{2} \ldots \mu_{d}} f_{\mu_{1} \mu_{2}}\right] .
$$

Now, a trivial shift $b+s \rightarrow b$ in the integration variable $b$ puts the source dependence into the constraint

$$
Z_{f e r}[s]=\int D b_{\mu} \operatorname{det}(\not+m+i \not) \delta\left[\epsilon_{\mu_{1} \mu_{2} \ldots \mu_{d}}\left(f_{\mu_{1} \mu_{2}}-2 \partial_{\mu_{1}} s_{\mu_{2}}\right)\right]
$$

It is at this point that the bosonic field, equivalent to the original fermion field, enters into play. Indeed, in order to handle the flatness condition, one represents the delta function in (9) in the form

$$
\begin{aligned}
& \left.\delta\left[\epsilon_{\mu_{1} \mu_{2} \ldots \mu_{d}}\left(f_{\mu_{1} \mu_{2}}-2 \partial_{\mu_{1}} s_{\mu_{2}}\right)\right)\right] \\
& =\int D \phi \exp \left[\frac{i}{2} \int d^{d} x \epsilon_{\mu_{1} \mu_{2} \ldots \mu_{d}} \phi_{\mu_{3} \ldots \mu_{d}}\left(f_{\mu_{1} \mu_{2}}-2 \partial_{\mu_{1}} s_{\mu_{2}}\right)\right]
\end{aligned}
$$

It is the Lagrange multiplier $\phi_{\mu_{3} \ldots \mu_{d}}$, which can be seen as a Kalb-Ramond field [17], that becomes the bosonic equivalent of the original fermion. Hence, the fermionic partition function can be finally written as

$$
\begin{aligned}
Z_{f e r}[s] & =\int D \phi \exp \left(-i \int d^{d} x \epsilon_{\mu_{1} \mu_{2} \ldots \mu_{d}}\left(\partial_{\mu_{2}} \phi_{\mu_{3} \ldots \mu_{d}}\right) s_{\mu_{1}}\right) \exp \left(-S_{b o s}[\phi]\right) \\
& \equiv Z_{b o s}[s]
\end{aligned}
$$

with

$$
\left.\exp \left(-S_{b o s}[\phi]\right)=\int D b_{\mu} \operatorname{det}(\not)+m+i \not\right) \exp \left(\frac{i}{2} \int d^{d} x \epsilon_{\mu_{1} \mu_{2} \ldots \mu_{d}} \phi_{\mu_{3} \ldots \mu_{d}} f_{\mu_{1} \mu_{2}}\right)
$$


The central point in our bosonization route is now at sight: the external source $s_{\mu}$ has factored out from the auxiliary field integration so that by simple differentiation one has the exact bosonization recipe for fermion currents

$$
\bar{\psi} \gamma_{\mu_{1}} \psi \rightarrow \epsilon_{\mu_{1} \mu_{2} \ldots \mu_{d}} \partial_{\mu_{2}} \phi_{\mu_{3} \ldots \mu_{d}}
$$

This, together with the recipe for bosonizing the fermion action

$$
\int \bar{\psi}(\not+m) \psi d^{3} x \rightarrow S_{b o s}[\phi]
$$

with $S_{\text {bos }}$ defined by eq.(12) completes the fermion-boson mapping in the Abelian case. Of course, one has still to perform the $b_{\mu}$ integration to give an explicit expression for $S_{\text {bos }}$. Whether one would arrive to a close result for the bosonized action or to an approximate one will depend on the possibility of computing the fermion determinant exactly and then performing the integration over $b$. This can be of course done in 2 dimensions where the well-known bosonization recipe can be derived within this approach in a very simple way [21]. If this were also possible for $d \geq 2$, then the fermionic degrees of freedom, which disappeared from the partition function, would be replaced by new bosonic degrees of freedom $\phi_{\mu_{3} \ldots \mu_{d}}$, in an exactly equivalent bosonic model. Even if one is not able to compute the fermion determinant exactly (as one does for $d=2$ ), it is important to note that the bosonization recipe for the fermion current is exact. It is the bosonic action that accompanies this recipe that has to be approximated.

\section{Current commutators}

We have seen in the precedent section that starting from the free fermion generating functional in $d$ dimensional space-time in the presence of a source $s_{\mu}$, one can systematically construct a bosonic theory and obtain in a very simple way the bosonization recipe for the fermionic current. We shall specialize in this section to 3-dimensional (Euclidean) space, so that the original fermionic generating functional.

$$
Z_{f e r}[s]=\int D \bar{\psi} D \psi \exp \left[-\int d^{3} x \bar{\psi}(\not \partial+m+i \not) \psi\right]
$$


becomes, after bosonization,

$$
\begin{aligned}
Z_{f e r}[s]= & \int D A_{\mu} \exp \left(-i \epsilon_{\mu \nu \alpha} \int d^{3} x s_{\mu} \partial_{\nu} A_{\alpha}\right) \times \\
& \int D b_{\mu} \operatorname{det}(\not \partial+m+i \not b) \exp \left(+i \epsilon_{\mu \nu \alpha} \int d^{3} x b_{\mu} \partial_{\nu} A_{\alpha}\right),
\end{aligned}
$$

where $A_{\mu}$ is the bosonic field $\left(A_{\mu}=\phi_{\mu}\right)$ replacing the original fermion field and $b_{\mu}$ is the auxiliary field to be integrated out.

As discussed in the precedent section, the possibility of going on with the bosonization procedure depends on one's ability to evaluate the fermion determinant in the r.h.s. of eq.(16) and then integrate out the auxiliary field $b_{\mu}$. In three dimensional space-time, one possibility is to use the results of the $1 / m$ expansion [31] to give an expression for the fermion determinant which will then be valid only for large masses. Alternatively, one can make an expansion in powers of $b_{\mu}$ retaining up to quadratic terms. Following this last procedure, integrating out $b_{\mu}$ and diagonalizing the resulting bosonic action, one ends with 22

$$
Z_{\text {fer }}[s]=Z_{b o s}[s]
$$

with

$$
\begin{aligned}
Z_{b o s}[s] & =\int D A_{\mu} \exp \left(-\int d^{3} x\left[\frac{1}{4} F_{\mu \nu} C_{1}\left(-\partial^{2}\right) F_{\mu \nu}\right.\right. \\
& -\frac{i}{2} A_{\mu} C_{2}\left(-\partial^{2}\right) \epsilon_{\mu \nu \lambda} \partial_{\nu} A_{\lambda} \\
& \left.\left.+i \frac{u_{+}-u_{-}}{2} s_{\mu} \frac{1}{\sqrt{-\partial^{2}}} \partial_{\nu} F_{\mu \nu}+i \frac{u_{+}+u_{-}}{2} s_{\mu} \epsilon_{\mu \nu \lambda} \partial_{\nu} A_{\lambda}\right]\right)
\end{aligned}
$$

Here $C_{1}$ and $C_{2}$ are defined as

$$
\begin{aligned}
C_{1} & =\frac{1}{2} \frac{\left|u_{+}\right|^{2}(F-i G)+\left|u_{-}\right|^{2}(F+i G)}{-\partial^{2} F^{2}+G^{2}} \\
C_{2} & =\frac{i}{2} \frac{\left|u_{+}\right|^{2}(F-i G)-\left|u_{-}\right|^{2}(F+i G)}{-\partial^{2} F^{2}+G^{2}}
\end{aligned}
$$

with $F\left(-\partial^{2}\right)$ and $G\left(-\partial^{2}\right)$ given through their momentum-space representations $\tilde{F}$ and $\tilde{G}$ as [22]

$$
\tilde{F}(k)=\frac{|m|}{4 \pi k^{2}}\left[1-\frac{1-\frac{k^{2}}{4 m^{2}}}{\left(\frac{k^{2}}{4 m^{2}}\right)^{\frac{1}{2}}} \arcsin \left(1+\frac{4 m^{2}}{k^{2}}\right)^{-\frac{1}{2}}\right],
$$




$$
\tilde{G}(k)=\frac{q}{4 \pi}+\frac{m}{2 \pi|k|} \arcsin \left(1+\frac{4 m^{2}}{k^{2}}\right)^{-\frac{1}{2}},
$$

Quantities $u_{ \pm}$are arbitrary functions of the momentum, arising in the diagonalization procedure. Concerning $q$, it is a regularization dependent parameter which can assume any integer value. Current correlation functions and, a fortiori, current commutators can be proven to be $u$-independent. For simplicity we shall take them equal and constant, $u_{ \pm}=u$ so that eq.(18) reduces to

$$
\begin{aligned}
Z_{b o s}= & \int D A_{\mu} \exp \left[-\int d^{3} x\left(\frac{1}{4} F_{\mu \nu} C_{1} F_{\mu \nu}-\frac{i}{2} A_{\mu} C_{2} \epsilon_{\mu \nu \lambda} \partial_{\nu} A_{\lambda}\right.\right. \\
& \left.\left.+i u s_{\mu} \epsilon_{\mu \nu \lambda} \partial_{\nu} A_{\lambda}\right)\right]
\end{aligned}
$$

with $C_{1}$ and $C_{2}$ now given through their momentum-space representation $\tilde{C}_{1}$ and $\tilde{C}_{2}$

$$
\begin{aligned}
& \tilde{C}_{1}(k)=|u|^{2} \frac{\tilde{F}(k)}{k^{2} \tilde{F}^{2}(k)+\tilde{G}^{2}(k)} \\
& \tilde{C}_{2}(k)=|u|^{2} \frac{\tilde{G}(k)}{k^{2} \tilde{F}^{2}(k)+\tilde{G}^{2}(k)}
\end{aligned}
$$

Equation (23) gives the bosonized version of the original fermion model partition function in the presence of an external source, in the quadratic approximation. We again see that the bosonization recipe advanced in [18] for the large mass case is in fact valid at all scales,

$$
\bar{\psi} \gamma_{\mu} \psi \rightarrow \frac{i}{\sqrt{4 \pi}} \epsilon_{\mu \nu \alpha} \partial_{\nu} A_{\alpha}
$$

(To compare with ref. [18] we have made the choice $u=i / \sqrt{4 \pi}$ ). The point is that the r.h.s. in eq.(26) involves a bosonic field with dynamics governed by the bosonic action

$$
S_{b o s}=\int\left[\frac{1}{4} F_{\mu \nu} C_{1}\left(-\partial^{2}\right) F_{\mu \nu}-\frac{i}{2} A_{\mu} C_{2}\left(-\partial^{2}\right) \epsilon_{\mu \nu \lambda} \partial_{\nu} A_{\lambda}\right] d^{3} x
$$

As discussed in detail in refs. 17] and 21], in the path-integral approach to bosonization, the bosonic field naturally appears as a vector field with a gauge invariant action (27) so that the corresponding partition function $Z_{\text {bos }}$ will require a gauge fixing. 
One can easily see that eqs.(23)-(27) reproduce, in the large mass limit, the bosonization result given in [18], [21]. Indeed, taking the limit of large masses in the expressions for $C_{1}$ and $C_{2}$ (eqs.(24)-(25)) one reobtains the derivative expansion result for the 3-dimensional fermion determinant [31[32] so that the generating functional (23) becomes within this approximation 22

$$
\begin{aligned}
Z_{b o s}[s] & \simeq \int D A_{\mu} \exp \left[-\int d^{3} x\left(\mp \frac{i}{2} \epsilon_{\mu \nu \alpha} A_{\mu} \partial_{\nu} A_{\alpha}+\frac{1}{12|m|} F_{\mu \nu}^{2}\right.\right. \\
& \left.\left.-\frac{1}{\sqrt{4 \pi}} \epsilon_{\mu \nu \alpha} s_{\mu} \partial_{\nu} A_{\alpha}\right)\right]
\end{aligned}
$$

(Again, to compare with 18 we have made $u=i /(\sqrt{4 \pi})$ and chosen the arbitrary parameter $q$ so that $q \pm m /|m|= \pm 1$.) From eq.(28) we see that the fermion system is described for large masses by a Maxwell-Chern-Simons theory. Now, the gauge invariant algebra of such theory has been studied in refs. 24], 26]. One has for instance in our case,

$$
\left[E_{i}(\vec{x}, t), B(\vec{y}, t)\right]=-3|m| \epsilon_{i j} \partial_{j} \delta^{(2)}(\vec{x}-\vec{y})
$$

If one now relates the electric field $E_{i}=F_{i 0}$ and the magnetic field $B=$ $\epsilon_{i j} \partial_{i} A_{j}$ to the fermionic currents through the bosonization recipe (11),

$$
\begin{gathered}
j_{o} \rightarrow \frac{1}{\sqrt{4 \pi}} B \\
j_{i} \rightarrow \frac{1}{\sqrt{4 \pi}} \epsilon_{i j} E_{j}
\end{gathered}
$$

then, the resulting fermion current commutator algebra is not the one to be expected for three-dimensional free fermions. Indeed, the $d=3$ fermion current algebra should contain an infinite Schwinger term [10]- [27] which is absent in eq.(29). The point is that eq.(28) ensuring that the resulting bosonic theory is a Maxwell-Chern-Simons theory, is valid only for large fermion mass while calculation of equal-time current commutators imply, as we shall see, a limiting procedure which cannot be naively followed for large masses.

Since the exact bosonic partition function is much too complicated to handle, a possible strategy is to use the quadratic (in auxiliary fields) approximation for fermions with an arbitrary (not necessarily large) mass so 
as to obtain a bosonized version of the original fermionic model in which the equal-time limit can be safely taken. One should then compute current commutators for this bosonized theory, and test whether they coincide with those satisfied by fermionic currents in the original model. As we shall see, our bosonization scheme does reproduce the correct equal time commutator algebra; moreover, although we find equal time commutators for arbitrary mass, it becomes apparent that the divergent Schwinger term can already be obtained in the small mass limit, where the bosonized action takes a reasonably simple form so that finally one disposes of a simple bosonization recipe covering both the small and large distances regimes. The recipe would be unique in the sense it gives a unique relation between the fermionic current and the bosonic field curl although the effective bosonic theory would differ in each regime.

To proceed according to this strategy, let us rewrite the partition function (23) (taking from here on $u=i / \sqrt{4 \pi}$ ) in the form

$$
\begin{aligned}
Z_{b o s}[s]= & \int D A_{\mu} \exp \left[-\frac{1}{2} \int d^{3} x d^{3} y A_{\mu}(x) D_{\mu \nu}(x, y) A_{\nu}(y)\right. \\
& \left.-\frac{1}{\sqrt{4 \pi}} \int d^{3} x A_{\lambda} \partial_{\nu} s_{\mu} \epsilon_{\mu \nu \lambda}\right]
\end{aligned}
$$

or

$$
Z_{b o s}[s]=\left[\operatorname{det} D_{\mu \nu}\right]^{-\frac{1}{2}} \exp \left[\frac{1}{8 \pi} \int d^{3} x d^{3} y \partial_{\nu} s_{\mu}(x) \epsilon_{\mu \nu \lambda} D_{\lambda \rho}^{-1}(x, y) \partial_{\sigma} s_{\tau}(y) \epsilon_{\rho \sigma \tau}\right]
$$

where $D_{\mu \nu}^{-1}$ is just the propagator of the bosonic action, which in the Lorentz gauge we adopt from here on reads

$$
D_{\mu \nu}^{-1}(x, y)=\int \frac{d^{3} k}{(2 \pi)^{3}}\left[P(k) g_{\mu \nu}+Q(k) k_{\mu} k_{\nu}+R(k) \epsilon_{\mu \nu \alpha} k_{\alpha}\right] \exp i k(x-y)
$$

with

$$
\begin{gathered}
P(k)=\frac{\tilde{C}_{1}(k)}{k^{2} \tilde{C}_{1}^{2}(k)+\tilde{C}_{2}^{2}(k)}=4 \pi \tilde{F}(k) \\
Q(k)=\frac{\tilde{C}_{1}(k)}{k^{2} \tilde{C}_{1}^{2}(k)+\tilde{C}_{2}^{2}(k)}\left(\frac{\tilde{C}_{2}(k)}{k^{2} \tilde{C}_{1}(k)}\right)^{2}
\end{gathered}
$$




$$
R(k)=\frac{\tilde{C}_{2}(k)}{k^{2}\left(k^{2} \tilde{C}_{1}^{2}(k)+\tilde{C}_{2}^{2}(k)\right)}
$$

Let us briefly recall how one can compute current commutators within the path-integral scheme using the so-called BJL method [28]-29], [8]. To this end we define the correlator

$$
G_{\mu \nu}(x, y)=\left.\frac{\delta^{2} \log Z_{b o s}[s]}{\delta s_{\mu}(x) \delta s_{\nu}(y)}\right|_{s=0}
$$

from which one can easily derive equal time current commutators using the relation

$$
<\left[j_{0}(\vec{x}, t), j_{i}(\vec{y}, t)\right]>=\lim _{\epsilon \rightarrow 0^{+}}\left[G_{0 i}(\vec{x}, t+\epsilon ; \vec{y}, t)-G_{0 i}(\vec{x}, t-\epsilon ; \vec{y}, t)\right]
$$

The current commutator evaluated using eqs.(38)-(39) corresponds to the bosonic partition function $Z_{b o s}[s]$ obtained in eq.(23). That is, eq.(39) gives the equal-time commutator for the bosonic currents $j_{\mu}=(1 / \sqrt{4 \pi}) \epsilon_{\mu \nu \alpha} \partial_{\nu} A_{\alpha}$. This result should then be compared with that arising in the original 3 dimensional fermionic model for which $j_{\mu}=-i \bar{\psi} \gamma_{\mu} \psi$ [27].

Starting from eqs.(33)-(34) and using the BJL method we get, after some calculations,

$$
G_{\mu \nu}(x, y)=-\frac{1}{4 \pi} \epsilon_{\mu \alpha \rho} \epsilon_{\nu \beta \sigma} \partial_{\alpha} \partial_{\beta} D_{\rho \sigma}^{-1}
$$

or

$$
G_{\mu \nu}(x, y)=\frac{1}{4 \pi} \int \frac{d^{3} k}{(2 \pi)^{3}}\left[P(k)\left(k^{2} g_{\mu \nu}-k_{\mu} k_{\nu}\right)+k^{2} R(k) \epsilon_{\mu \nu \alpha} k_{\alpha}\right] \exp [i k(x-y)]
$$

With this, we can rewrite eq.(39) in the form

$$
<\left[j_{0}(\vec{x}, t), j_{i}(\vec{y}, t]>=\lim _{\epsilon \rightarrow 0^{+}} I^{\epsilon}(\vec{x}-\vec{y})\right.
$$

with

$$
I^{\epsilon}(\vec{x})=-2 i \int \frac{d^{3} k}{(2 \pi)^{3}} k_{0} k_{i} \sin \left(k_{0} \epsilon\right) \tilde{F}(k) \exp i \vec{k} \cdot \vec{x}
$$

where we have written $\left(k_{\mu}\right)=\left(k_{o}, k_{i}\right), i=1,2$. It will be convenient to define

$$
k_{0}^{\prime}=\epsilon k_{0}
$$


In terms of this new variable and using the explicit form for $\tilde{F}(k)$ given by eq.(21), with $k=\left(k_{0}^{2}+\vec{k}^{2}\right)^{1 / 2}$, integral $I^{\epsilon}$ becomes

$$
I^{\epsilon}(\vec{x})=-\frac{1}{8 \pi^{2}|m|} \frac{1}{\epsilon^{2}} \partial_{i} \int \frac{d^{2} k}{(2 \pi)^{2}} \exp i \vec{k} \cdot \vec{x} \int_{0}^{\infty} d k_{0}^{\prime} k_{0}^{\prime} \sin k_{0}^{\prime} f(y)
$$

where

$$
f(y)=\frac{1}{y}\left[1-\frac{(1-y)}{\sqrt{y}} \arcsin \frac{1}{\sqrt{1+(1 / y)}}\right]
$$

and we have defined

$$
y=\frac{k^{2}}{4 m^{2}}=\frac{k_{0}^{\prime 2}+\epsilon^{2} \vec{k}^{2}}{4 \epsilon^{2} m^{2}}
$$

One can now see that $y \rightarrow \infty$ for $\epsilon \rightarrow 0$ and fixed $m$. Then, expanding in powers of $1 / y$ one has $f(y) \sim \pi /(2 \sqrt{y})$ and then using distribution theory to define the integral over $k_{0}^{\prime}$ one finds

$$
<\left[j_{0}(\vec{x}, t), j_{i}(\vec{y}, t]>=-\frac{1}{8 \pi} \lim _{\epsilon \rightarrow 0} \frac{1}{\epsilon} \partial_{i} \delta^{(2)}(\vec{x}-\vec{y})\right.
$$

This result for the equal-time current commutator, evaluated within the bosonized theory, shows exactly the infinite Schwinger term that is found, using the BJL method, for free fermions in $d=3$ dimensions [27]. As it happens in $d=4$ dimensions [9]-11], we see from eq.(48) that the commutator at unequal times is well defined: divergencies appear only when one takes the equal-time limit.

It is interesting to evaluate the next order vanishing in the equal-time limit so as to compare with the result for the original fermion model reported in the literature [27]. Due to the combination $\epsilon m$ appearing in $y$ one can make $m \rightarrow 0$ in the equal-time limit without loss of generality. Then, one can first consider the limit of small mass, expand $f$, integrate exactly over $k_{0}^{\prime}$ and then take $\epsilon \rightarrow 0$. Now, for small mass one has

$$
f(y)=\pi|m|\left(\frac{1}{\left(\vec{k}^{2}+\left(k_{0}^{\prime} / \epsilon\right)^{2}\right)^{1 / 2}}-4 m^{2} \frac{1}{\left(\vec{k}^{2}+\left(k_{0}^{\prime} / \epsilon\right)^{2}\right)^{3 / 2}}+\ldots\right)
$$

so that, after integrating over $k_{0}^{\prime}$ one has, for $I^{\epsilon}(\vec{x})$,

$$
I^{\epsilon}(\vec{x})=-\frac{1}{8 \pi} \partial_{i} \int \frac{d^{2} k}{(2 \pi)^{2}} \exp i \vec{k} \cdot \vec{x}\left(|\vec{k}| K_{1}(\epsilon|\vec{k}|)-4 m^{2} \epsilon K_{0}(\epsilon|\vec{k}|)+\ldots\right)
$$


Using the modified Bessel functions expansion for small $\epsilon$ one easily gets

$$
\begin{aligned}
<\left[j_{0}(\vec{x}, t), j_{i}(\vec{y}, t]>=\right. & \lim _{\epsilon \rightarrow 0}\left[-\frac{1}{8 \pi} \frac{1}{\epsilon}\left(1+4 m^{2} \epsilon^{2} \log \epsilon\right) \times \partial_{i} \delta^{(2)}(\vec{x}-\vec{y})+\right. \\
& \left.\frac{1}{16 \pi} \epsilon \log \epsilon \partial_{i} \Delta \delta^{(2)}(\vec{x}-\vec{y})\right]
\end{aligned}
$$

The second and third terms in eq.(51), which vanish in the equal-time limit, are precisely the 3 dimensional analogs of those encountered in $d=4$ dimensions [9]-[11]. Now, to compare our results with those reported in [27] using dimensional regularization, we shall expand $f(y)$ in eq.(49) in powers of $\epsilon$ (keeping the mass fixed) before integrating out $k_{0}^{\prime}$. In this way, one ends with

$$
\begin{aligned}
<\left[j_{0}(\vec{x}, t), j_{i}(\vec{y}, t]>=\right. & -\frac{1}{8 \pi} \lim _{\epsilon \rightarrow 0}\left(\frac{1}{\epsilon} \partial_{i} \delta^{(2)}(\vec{x}-\vec{y})\right. \\
& \left.-\frac{\epsilon}{\Lambda}\left[4 m^{2} \partial_{i} \delta^{(2)}(\vec{x}-\vec{y})-\frac{1}{2} \partial_{i} \Delta \delta^{(2)}(\vec{x}-\vec{y})\right]\right)
\end{aligned}
$$

where we have defined

$$
\frac{1}{\Lambda}=\int_{0}^{\infty} d k_{0}^{\prime} \frac{1}{k_{0}^{\prime 2}} \sin k_{0}^{\prime}
$$

In order to compare with ref. [27] where current commutators were computed using dimensional regularization, we define, coming back to the original variable $k_{0}=k_{0}^{\prime} / \epsilon$

$$
A[d]=\frac{1}{2} \int d^{d-2} k_{0} \frac{1}{k_{0}^{2}} \sin k_{0} \epsilon
$$

so that $A[d=3]=\epsilon / \Lambda$. One can now perform the analytically continued integral to find, near $d=3$, the behavior

$$
A[d] \sim-\epsilon \times \frac{\epsilon^{3-d}}{3-d}
$$

The same ambiguous result eqs.(52),(55) for free fermions is obtained in ref. [27] near $d=3$. This ambiguity is however removed by our previous procedure leading to eq.(51), the pole in dimensional regularization corresponding as usual to a logarithmic divergence. It is also interesting to note that using in eq.(43) the nice approximation $\tilde{F}_{\text {appr }}$ for $\tilde{F}$ proposed in ref. [22], one can well check the correctness of our previous analysis. In fact, on the 
one hand both procedures leading to either eq.(51) or eq.(52) give (apart for the coefficient of $m^{2}$ ) the same result using $\tilde{F}_{\text {appr }}$ or $\tilde{F}$. But, on the other hand, for arbitrary mass, the the $k_{0}^{\prime}$ integration can be easily done exactly if one uses $\tilde{F}_{\text {appr }}$. The subsequent $\epsilon \rightarrow 0$ limit gives precisely (again apart for the coefficient of $\mathrm{m}^{2}$ ) our result (51) for the equal-time current commutators.

From the analysis above, we see that not only the infinite Schwinger term, analogous to that arising in $d=4$ [9]-11] is obtained in the bosonized version of our $d=3$ fermion theory but also the mass-dependent second term as well as the triple derivative third term in (51), both vanishing in the equal time limit.

We then conclude that the equal-time commutator algebra for bosonic currents $j_{\mu}=(1 / \sqrt{4 \pi}) \epsilon_{\mu \nu \alpha} \partial_{\nu} A_{\alpha}$ coincides with that for fermion currents, $j_{\mu}=-i \bar{\psi} \gamma_{\mu} \psi$. It is interesting to note at this point that the $m \rightarrow 0$ theory has the same equal time current commutators that the theory for arbitrary mass. Now, for the massless limit one obtains a very simple bosonized action. Indeed, for $m \rightarrow 0$ one has from the expressions (21)-(22), (24)-(25)

$$
\begin{gathered}
\tilde{C}_{1}=\frac{4}{\pi k} \sin ^{2} \alpha \\
\tilde{C}_{2}=\frac{k}{\tan \alpha} \tilde{C}_{1} \\
\tilde{F}=\frac{1}{16 k}
\end{gathered}
$$

with $\tan \alpha=(\pi / 4 q)$ so that the bosonized action takes the form 22

$$
S_{\text {bos }}=\frac{4}{\pi} \sin ^{2} \alpha \int d^{3} x\left[\frac{1}{4} F_{\mu \nu} \frac{1}{\sqrt{-\partial^{2}}} F_{\mu \nu}-\frac{i}{2} \cot \alpha \epsilon_{\mu \nu \lambda} A_{\mu} \partial_{\nu} A_{\lambda}\right]
$$

which is nothing but the action found in 13] for free massless fermions. We thus see that our bosonized effective theory correctly describes the commutator algebra directly computed from the original fermionic theory. This should be compared with the analysis in ref. 30] where the fermionic commutator algebra is inferred from the Maxwell-Chern-Simons algebra for electric and magnetic fields using a bosonization recipe which is valid in the large mass limit. One can see that in the large mass regime, terms depending on the product $\epsilon m=\lambda$ will produce ambiguities according to the way both limits 
$(\epsilon \rightarrow 0$ and $m \rightarrow \infty)$ are taken into account, a problem which is not present in the limit of small masses. To see this in more detail, let us come back to (43) and consider the case in which $\lambda$ is kept fixed while $\epsilon \rightarrow 0$ (so that $m \rightarrow \infty)$. In this case, taking the limit before integrating out $k_{0}^{\prime}$, one finds for $I^{\epsilon}$

$$
I^{\epsilon}(\vec{x}) \sim|m| h(\lambda) \partial_{i} \delta^{(2)}(\vec{x})
$$

where

$$
h(\lambda)=\frac{1}{2 \pi} \int_{0}^{\infty} d z z \sin (2 \lambda z) f(z)
$$

with $f$ given by eq.(46). Let us note that using the approximate $\tilde{F}_{\text {appr }}$ of ref. 22 and taking the limit after the exact integration over $k_{0}^{\prime}$, we recover the same behavior (60). We see that for $\lambda=\epsilon m$ fixed, $h$ just gives a normalization factor so that one reproduces from $I^{\epsilon}$ in the form (60) a commutator algebra at equal times and large mass that coincides with that to be infered from a Maxwell-Chern-Simons theory, namely (cf. eqs.(29)-(31))

$$
<\left[j_{0}(\vec{x}, t), j_{i}(\vec{y}, t]>\longrightarrow c|m| \partial_{i} \delta^{(2)}(\vec{x}-\vec{y}) \quad(m \rightarrow \infty)\right.
$$

with $c$ a normalization constant. Again, currents appearing in the l.h.s. of eq. (62) are bosonic currents which can be written in terms of the electric and magnetic fields thus reproducing the MCS gauge invariant algebra [24], [26]. One should note however that the free fermion - MCS mapping is valid in the large mass limit of the original fermionic theory, this meaning the largedistances regime for fermion fields. Since current commutators test the shortdistance regime, one should not take the MCS gauge-invariant algebra as a starting point to reproduce the fermion current commutators.

\section{Summary and conclusions}

As explained at length in the precedent sections, one can establish in $d=3$ dimensional space-time a bosonization recipe for fermion currents which is the natural extension of the 2 dimensional one. Namely, one has (apart from normalization factors)

$$
-i \bar{\psi} \gamma_{\mu} \psi \rightarrow \frac{1}{\sqrt{4 \pi}} \epsilon_{\mu \nu \alpha} \partial_{\nu} A_{\alpha}, \quad(d=3)
$$


to be compared with the well-known recipe

$$
-i \bar{\psi} \gamma_{\mu} \psi \rightarrow \frac{1}{\sqrt{4 \pi}} \epsilon_{\mu \nu} \partial_{\nu} \phi, \quad(d=2)
$$

One can see eq.(63), which gives an exact bosonization recipe, as the natural extension to three dimensional space of the two-dimensional formula (64). It is the bosonized action that is much more complicated in 3 dimensions. Instead of a simple scalar action as in 2 dimensions, one has (in a quadratic approximation in the auxiliary fields)

$$
\int \bar{\psi}(\not \partial+m) \psi d^{3} x \rightarrow \int\left[\frac{1}{4} F_{\mu \nu} C_{1}\left(-\partial^{2}\right) F_{\mu \nu}-\frac{i}{2} A_{\mu} C_{2}\left(-\partial^{2}\right) \epsilon_{\mu \nu \lambda} \partial_{\nu} A_{\lambda}\right] d^{3} x
$$

with $C_{1}$ and $C_{2}$ given by eqs.(19)-(22). Only in the limit of very large and very small fermion mass the bosonized action takes a simple form. As shown by eq.(28) and in ref. [22, the bosonization relation (65) becomes for large fermion mass the Maxwell-Chern-Simons action [18 -21

$$
\begin{aligned}
\int \bar{\psi}(D+m) \psi d^{3} x \longrightarrow & \int\left[\frac{1}{12|m|} F_{\mu \nu} F_{\mu \nu}\right. \\
& \left.\mp \frac{i}{2} A_{\mu} \epsilon_{\mu \nu \lambda} \partial_{\nu} A_{\lambda}\right] d^{3} x \quad(m \rightarrow \infty)
\end{aligned}
$$

On the other hand, for $m \rightarrow 0$ and in a quadratic approximation the action becomes (see eq.(59))

$$
\begin{aligned}
\int \bar{\psi}(\not+m) \psi d^{3} x \longrightarrow & \frac{4}{\pi} \sin ^{2} \alpha \int\left[\frac{1}{4} F_{\mu \nu} \frac{1}{\sqrt{-\partial^{2}}} F_{\mu \nu}\right. \\
& \left.-\frac{i}{2} \cot \alpha \epsilon_{\mu \nu \lambda} A_{\mu} \partial_{\nu} A_{\lambda}\right] d^{3} x \quad(m \rightarrow 0)
\end{aligned}
$$

Either recipe (66) or (67) should be used according to the regime one is to analyse. For example, in the study of Wilson loops in the long distance region, one employs relation (66) finding an interesting connection between the linking number and certain fermion loop operators [18]. Concerning massless fermions one should use eq. 67) which coincides (apart from normalization factors) to that proposed in ref. [13], eq.(59).

In respect with current commutators, we have shown in this work that the bosonization recipe (63),(65) reproduces the equal time current commutator algebra corresponding to the original 3-dimensional free massive fermion 
model for arbitrary mass,

$$
<\left[j_{0}(\vec{x}, t), j_{i}(\vec{y}, t]>=-\frac{1}{8 \pi} \lim _{\epsilon \rightarrow 0} \frac{1}{\epsilon} \partial_{i} \delta^{(2)}(\vec{x}-\vec{y})\right.
$$

with currents in the 1.h.s. of eq.(68) expressed in terms of the bosonic field according to eq.(63). As explained, this result can be obtained even if one works in the $m \rightarrow 0$ limit and uses bosonization recipes (63),(67) which implies a simpler bosonic action. On the other hand, the finite Schwinger term one finds starting directly from eq. 66) is just the product of the way one takes the equal-time and the $m \rightarrow \infty$ limits. However, since the $m \rightarrow \infty$ limit explores the large distances regime of the fermion theory, one should not use the MCS gauge-invariant algebra to reproduce the fermion current commutators which test short distances.

In summary, the path-integral approach to bosonization has shown to give a general bosonization recipe for free-fermions in 3 space-time dimensions leading to the correct equal-time current commutation algebra. This implies a step further in the obtention of the fermion-boson mapping in $d=3$. As explained in refs. [18, 20] 21], this method is particularly apt to treat interacting and non-Abelian extensions. Moreover, the analysis of the $d>3$ case can be envisaged following a similar approach. We hope to report on these issues in a future work.

Acknowledgements: We would like to thank R. Jackiw for very useful comments that prompted this paper. This work was supported in part by CICBA and CONICET, Argentina and the Ministère de l'Enseignement Supérieure et de la Recherche (France). F.A.S. thanks the Laboratoire de Physique Théorique ENSLAPP for its kind hospitality.

\section{References}

[1] E. Lieb and D. Mattis, J. Math. Phys. 6 (1965), 304.

[2] A. Luther and I. Peschel, Phys. Rev. B 9 (1974), 2811.

[3] S. Coleman, Phys. Rev. D 11 (1975), 2088.

[4] S. Mandelstam, Phys. Rev. D 11 (1975), 3026. 
[5] E. Witten,Comm. Math. Phys. 92 (1984), 455.

[6] T. Goto and T. Imamura, Progr. Theoret. Phys. (Kyoto) 14 (1955), 396.

[7] J. Schwinger, Phys. Rev. Lett. 3 (1959), 1795.

[8] For a review see. e.g. R.Jackiw in "Lectures on Current Alebra and its Applications", (eds. S.B. Treiman, R. Jackiw and D.J. Gross), Princeton University Press, 1972.

[9] R. Brandt,Phys. Rev. 166 (1968), 1795.

[10] D.G. Boulware and R. Jackiw, Phys. Rev. 186 (1969), 1442.

[11] M.S. Chanowitz, Phys. Rev. D 2 (1970) 3016; D 4 (1971), 1717.

[12] A. Luther, Phys. Rev. D 19 (1979), 320.

[13] E.C. Marino, Phys. Lett. B 263 (1991), 63.

[14] F.D.M. Haldane, Helv. Phys. Acta 65 (1992), 52.

[15] A. Kovner and P.S. Kurzepa, Phys. Lett. B 321 (1994), 129.

[16] C.P. Burgess and F. Quevedo, Nucl. Phys. B 421 (1994), 373.

[17] C.P. Burgess, C.A. Lütken and F. Quevedo, Phys. Lett. B 336 (1994), 18.

[18] E. Fradkin and F.A. Schaposnik, Phys. Lett. B 338 (1994), 253.

[19] J. Frohlich, R. Götschmann and P.A. Marchetti, J.Phys. A28 (1995), 1169.

[20] N. Bralic, E. Fradkin, M.V. Manías and F.A. Schaposnik, Nucl. Phys. B (1995)

[21] F.A. Schaposnik,Phys. Lett. B 356 (1995), 39.

[22] D.G. Barci, C.D. Fosco and L.E. Oxman, "On bosonization in 3 dimensions" hep-th/9508075 report (unpublished). 
[23] Th. Jolicoeur and J.C. Le Guillou, Int. Jour. of Mod. Phys. A8 (1993), 1923.

[24] S. Deser, R. Jakiw and S. Templeton, Phys. Rev. Lett. 48 (1982) 975; Ann. of Physics (N.Y) 140 (1982), 372.

[25] See, e.g., E.Fradkin, "Field Theories of Condensed Matter Physics", Frontiers in Physics, New York, 1991, and references therein.

[26] S. Deser and R. Jackiw, Phys. Lett. B139 (1984), 371.

[27] H.J. de Vega and H.O. Girotti, Nucl. Phys. B 79 (1974), 77.

[28] J.D. Bjorken, Phys. Rev. 148 (1966) 1467.

[29] K. Johnson and F.E. Low, Prog. Theoret. Phys. (Kyoto), Suppl. 37-38 (1966), 74 .

[30] R. Banerjee, Phys. Lett. B 358 (1995), 297.

[31] A.N. Redlich, Phys. Rev Lett. 52 (1984), 18; Phys. Rev. D 29 (1984), 2366 .

[32] A.N. Redlich and S. Deser, Phys. Rev. Lett. 61 (1988), 1541. 